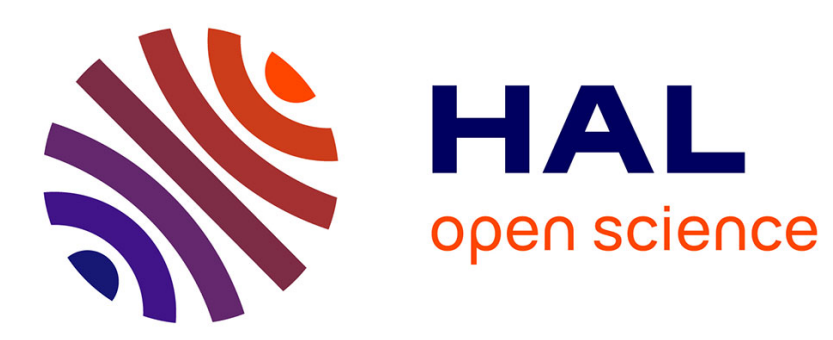

\title{
Fluctuations de charge et de masse dans un sel fondu binaire a la limite hydrodynamique
}

\author{
P. Vieillefosse
}

\section{To cite this version:}

P. Vieillefosse. Fluctuations de charge et de masse dans un sel fondu binaire a la limite hydrodynamique. Journal de Physique Lettres, 1977, 38 (2), pp.43-46. 10.1051/jphyslet:0197700380204300 . jpa-00231321

\section{HAL Id: jpa-00231321 https://hal.science/jpa-00231321}

Submitted on 1 Jan 1977

HAL is a multi-disciplinary open access archive for the deposit and dissemination of scientific research documents, whether they are published or not. The documents may come from teaching and research institutions in France or abroad, or from public or private research centers.
L'archive ouverte pluridisciplinaire HAL, est destinée au dépôt et à la diffusion de documents scientifiques de niveau recherche, publiés ou non, émanant des établissements d'enseignement et de recherche français ou étrangers, des laboratoires publics ou privés. 


\title{
FLUCTUATIONS DE CHARGE ET DE MASSE DANS UN SEL FONDU BINAIRE A LA LIMITE HYDRODYNAMIQUE
}

\author{
P. VIEILLEFOSSE
}

Laboratoire de Physique Théorique des Liquides (*)

Université Pierre-et-Marie-Curie, 4, place Jussieu, 75230 Paris Cedex 05, France

(Reçu le 14 octobre 1976, accepté le 2 décembre 1976)

\begin{abstract}
Résumé. - Nous montrons, par une étude hydrodynamique, que les sels fondus binaires se comportent essentiellement comme un fluide simple non chargé. Une simulation de dynamique moléculaire récente avait mis en évidence un mode propre optique, correspondant à des oscillations de charge. Seul, l'amortissement de ces oscillations, dû à l'interdiffusion, est observé à la limite hydrodynamique. Par contre, le son qui n'apparaissait pas dans l'étude de dynamique moléculaire est mis en évidence ici.
\end{abstract}

Abstract. - We show, by a hydrodynamic calculation, that binary molten salts behave essentially like simple neutral liquids. A recent molecular dynamics simulation exhibited an optic mode, due to charge density oscillations. The hydrodynamic study leads only to the damping of this mode, due to interdiffusion, and to a sound mode, which had not been observed in the computer simulation.

1. Introduction. - Notre étude des systèmes, composés de deux espèces ioniques de signes opposés, généralise celle faite pour les systèmes binaires non coulombiens par Mountain et Deutch [1] et celle faite pour le plasma à un composant (P.U.C.) [2]. Nous étudierons, dans une première partie, les fluctuations thermodynamiques, et, dans une deuxième partie, nous regarderons évoluer une telle fluctuation, suivant les équations de l'hydrodynamique linéarisée; ceci nous permettra d'atteindre le comportement des fonctions de corrélation de charge et de masse, à la limite des petits vecteurs d'onde $k$ et des petites fréquences $\omega$.

Le résultat essentiel de cette étude est la disparition du mode optique ou plasmon, observé en dynamique moléculaire par Hansen et Mc Donald [3].

2. Fluctuations thermodynamiques. - La description thermodynamique d'un système à deux composants se fait avec trois variables; nous prendrons la température $T$, la densité de charge $\rho_{0}$, et la densité de masse $\rho_{m}$. La différentielle de l'énergie interne par unité de volume $\varepsilon$ s'écrit alors sous la forme :

$$
\delta \varepsilon=T \delta \sigma+\mu_{0} \delta \rho_{0}+\mu_{m} \delta \rho_{m}
$$

où $\sigma$ est l'entropie par unité de volume, et $\mu_{0}$ et $\mu_{m}$ sont des potentiels chimiques. Nous en déduisons la variation de l'entropie par unité de volume, au second ordre, d'une façon analogue au cas du P.U.C. [2].

$$
\delta \sigma=\frac{1}{T} \delta \varepsilon-\frac{\mu_{0} \delta \rho_{0}+\mu_{m} \delta \rho_{m}}{T}+\frac{1}{2}\left\{\delta\left(\frac{1}{T}\right) \delta \varepsilon-\delta\left(\frac{\mu_{0}^{\prime}}{T}\right) \delta \rho_{0}-\delta\left(\frac{\mu_{m}}{T}\right) \delta \rho_{m}\right\} .
$$

Intégrant sur le volume du système et passant en série de Fourier (le système est supposé répété périodiquement dans l'espace), nous obtenons pour la variation de l'entropie totale $\delta S$ :

$$
\begin{aligned}
\delta S=-\frac{\rho_{m}}{2 T} \frac{1}{V} \sum_{k} v_{k} \cdot v_{-k}-\frac{C_{\mathrm{v}}}{2 T^{2}} \frac{1}{V} \sum_{k}\left|\delta T_{k}\right|^{2}- & \\
& -\frac{1}{2 T V} \sum_{k \neq 0}\left\{\frac{4 \pi e^{2}}{k^{2}}+\left.\frac{\partial \mu_{0}}{\partial \rho_{0}}\right|_{T \rho_{m}}\left|\delta \rho_{0 k}\right|^{2}+\left.2 \frac{\partial \mu_{0}}{\partial \rho_{m}}\right|_{T \rho_{0}} \delta \rho_{0 k} \delta \rho_{m-k}+\left.\frac{\partial \mu_{m}}{\partial \rho_{m}}\right|_{T \rho_{0}}\left|\delta \rho_{m k}\right|^{2}\right\}
\end{aligned}
$$

$\left(^{*}\right)$ Equipe Associée au C.N.R.S. 
où $C_{\mathrm{v}}$ est la chaleur spécifique par unité de volume $\left(T \partial \sigma / \partial T \mid \rho_{0} \rho_{m}\right)$. Nous avons tenu compte de la conservation de la charge totale, de la masse totale et de l'énergie totale, à savoir que la variation d'énergie interne était l'opposé de la somme de l'énergie cinétique, associée à la vitesse macroscopique $v$, et de l'énergie potentielle électrique, associée à la densité de charge macroscopique $\delta \rho_{0}$.

La probabilité d'avoir une fluctuation thermodynamique décrite par $V_{k}, \delta T_{k}, \delta \rho_{0 k}$ et $\delta \rho_{m k}$ est proportionnelle à $\mathrm{e}^{\delta S / k_{\mathrm{B}}}$, où $k_{\mathrm{B}}$ est la constante de Boltzmann. Nous en déduisons les expressions des facteurs de structure de charge et de masse, à la limite des petits vecteurs d'onde $k$ :

$$
\begin{aligned}
& S_{0}(k)=\frac{1}{V}\left\langle\delta \rho_{0 k} \delta \rho_{0-k}\right\rangle \underset{k \rightarrow 0}{\simeq} k_{\mathrm{B}} T \frac{\left.\frac{\partial \mu_{m}}{\partial \rho_{m}}\right|_{T \rho_{0}}}{\left.\left(\frac{4 \pi e^{2}}{k^{2}}+\left.\frac{\partial \mu_{0}}{\partial \rho_{0}}\right|_{T \rho_{m}}\right) \frac{\partial \mu_{m}}{\partial \rho_{m}}\right|_{T \rho_{0}}-\left[\left.\frac{\partial \mu_{0}}{\partial \rho_{m}}\right|_{T \rho_{0}}\right]^{2}} \\
& S_{m}(k)=\frac{1}{V}\left\langle\delta \rho_{m k} \delta \rho_{m-k}\right\rangle \underset{k \rightarrow 0}{\simeq} k_{\mathrm{B}} T \frac{\frac{4 \pi e^{2}}{k^{2}}+\left.\frac{\partial \mu_{0}}{\partial \rho_{0}}\right|_{T \rho_{m}}}{\left.\left(\frac{4 \pi e^{2}}{k^{2}}+\left.\frac{\partial \mu_{0}}{\partial \rho_{0}}\right|_{T \rho_{m}}\right) \frac{\partial \mu_{m}}{\partial \rho_{m}}\right|_{T \rho_{0}}-\left[\left.\frac{\partial \mu_{0}}{\partial \rho_{m}}\right|_{T \rho_{0}}\right]^{2}}
\end{aligned}
$$

et le terme de couplage charge-masse

$$
\frac{1}{V}\left\langle\delta \rho_{0 k} \delta \rho_{m-k}\right\rangle \underset{k \rightarrow 0}{\simeq} k_{\mathrm{B}} T \frac{-\left.\frac{\partial \mu_{0}}{\partial \rho_{m}}\right|_{T \rho_{0}}}{\left.\left(\frac{4 \pi e^{2}}{k^{2}}+\left.\frac{\partial \mu_{0}}{\partial \rho_{0}}\right|_{T \rho_{m}}\right) \frac{\partial \mu_{m}}{\partial \rho_{m}}\right|_{T \rho_{0}}-\left[\left.\frac{\partial \mu_{0}}{\partial \rho_{m}}\right|_{T \rho_{0}}\right]^{2}} .
$$

Dans la suite nous étudierons essentiellement le cas le plus simple de sel fondu, celui où l'Hamiltonien est totalement symétrique dans la permutation globale des deux espèces ioniques; en particulier les masses et les valeurs absolues des charges des anions et cations sont égales. Nous appellerons un tel sel fondu symétrique, SFS, dans la suite. Il est facile de voir, en explicitant la valeur moyenne canonique, que la symétrie de l'Hamiltonien entraîne que les fluctuations de charge et de masse sont indépendantes

$$
\left\langle\delta \rho_{0 k} \delta \rho_{m-k}\right\rangle=0 ;
$$

nous en déduisons que pour le SFS :

$$
\left.\frac{\partial \mu_{0}}{\partial \rho_{m}}\right|_{T \rho_{0}}=0 .
$$

La stabilité du système exige que la forme quadratique $\delta S$ soit positive ; nous en déduisons, en particulier, que $\partial \mu_{m} / \partial \rho_{m} \mid T \rho_{0}>0$, ce qui donne pour le SFS, compte tenu de (7), $\partial P / \partial \rho_{m} \mid T \rho_{0}>0$ (P est la pression). Par contre $\partial \mu_{0} / \partial \rho_{0} \mid T \rho_{m}$ égal à $\rho_{0}^{-1} \partial P / \partial \rho_{0} \mid T \rho_{m}$ pour le SFS, n'a pas de signe déterminé.

3. Hydrodynamique. - Nous allons écrire quatre équations de conservation pour la masse, la charge, l'impulsion et l'énergie. Ces équations feront intervenir des courants dissipatifs liés aux gradients des différentes grandeurs, qui sont uniformes à l'équilibre.
Nous écrirons l'expression générale de ces courants, de sorte que la loi de croissance de l'entropie soit bien respectée [4]. Nous en déduirons le comportement dynamique d'un tel système à la limite des petits $k$ et $\omega$.

L'équation de conservation de la masse est

$$
\partial_{t} \rho_{m}+\operatorname{div} j_{m}=0
$$

où $j_{m}$ est le courant de masse, égal à $\rho_{m} v$ par définition de la vitesse macroscopique $v$. Pour la charge nous avons :

$$
\partial_{t} \rho_{0}+\operatorname{div} j_{0}=0
$$

pour l'impulsion

$$
\partial_{t} j_{m i}+\partial_{k} \pi_{i k}=0
$$

pour l'énergie

$$
\partial_{t}\left(\varepsilon+\frac{\rho_{m} v^{2}}{2}+\frac{E^{2}}{8 \pi}\right)+\operatorname{div} j_{\varepsilon}=0
$$

$E^{2} / 8 \pi$ est l'énergie potentielle électrique associée au champ électrique $E$ de la densité de charge $\rho_{0}$.

Passons maintenant aux expressions des courants. Les courants comprennent. éventuellement, une contribution d'équilibre (par exemple la pression dans le tenseur de courant d'impulsion), une contribution liée au mouvement macroscopique du fluide, et enfin une contribution de diffusion liée aux différents gradients. Nous sommes ainsi conduits à écrire : 


$$
\begin{aligned}
& j_{0}=\rho_{0} v+j_{0 \text { diff. }} \\
& \pi_{i k}=P \delta_{i k}-\frac{1}{4 \pi}\left(E_{i} E_{k}-\frac{1}{2} E_{l}^{2} \delta_{i k}\right)+\rho_{m} v_{i} v_{k}-\sigma_{i k}^{\prime} \\
& j_{\varepsilon i}=\left(\varepsilon+P+\rho_{m} \frac{v^{2}}{2}\right) v_{i}-v_{k} \sigma_{i k}^{\prime}+q_{i} .
\end{aligned}
$$

Ces équations définissent les courants dissipatifs $j_{0 \text { diff. }}, \sigma^{\prime}$ et $q$.

Les équations (8) à (11) permettent de calculer le taux d'accroissement de l'entropie par unité de volume, dû aux processus de diffusion :

$$
\mathrm{d}_{t} \sigma=\frac{1}{T} \sigma_{i k}^{\prime} \partial_{k} v_{i}+\left(q-\mu_{0} j_{0 \text { diff. }}\right) \cdot \nabla \frac{1}{T}-\frac{1}{T} j_{0 \text { diff. }} \nabla\left(\mu_{0}+e \varphi\right)
$$

dans cette équation $\varphi$ est le potentiel électrostatique associé à $E(=-\nabla \varphi)$. Le membre de droite de (15) doit être essentiellement positif ; ceci nous conduit, en appliquant les règles habituelles [4], aux expressions suivantes des courants dissipatifs :

$$
\begin{gathered}
\sigma_{i k}^{\prime}=\eta\left(\partial_{k} v_{i}+\partial_{i} v_{k}-\frac{2}{3} \delta_{i k} \partial_{l} v_{l}\right)+\varphi \delta_{i k} \partial_{l} v_{l} \\
j_{0 \text { diff. }}=-\alpha \nabla\left(\mu_{0}+e \varphi\right)-\beta \nabla T \\
q-\mu_{0} j_{0 \text { diff. }}=-\beta T \nabla\left(\mu_{0}+e \varphi\right)-\gamma \nabla T
\end{gathered}
$$

La conductivité thermique est alors $\chi=\gamma-\beta^{2} T / \alpha$. De plus nous avons les conditions suivantes :

$$
\eta>0, \quad \varphi>0, \quad \alpha>0, \quad \gamma>0, \quad \chi>0 .
$$

Les coefficients cinétiques introduits permettent en principe de résoudre complètement le problème de l'écoulement hydrodynamique du fluide. Nous nous intéressons à la résorption d'une petite fluctuation et considérons le système d'équations linéarisées. Des résultats plus complets seront publiés ultérieurement ; nous ne donnons ici que les plus importants et pour le SFS. La méthode employée est la même que pour le P.U.C. [2].

Un tel système présente trois modes propres hydrodynamiques. Le premier est un mode de diffusion de charge avec une fréquence :

$$
\omega=-i 4 \pi e^{2} \alpha .
$$

Le second est un mode sonore amorti :

$$
\omega= \pm k \sqrt{\left.\frac{\partial P}{\partial \rho_{m}}\right|_{\sigma \rho_{0}}}-i \frac{k^{2}}{2}\left\{\frac{\frac{4}{3} \eta+\varphi}{\rho_{m}}+\frac{\chi}{\left.T \frac{\partial \sigma}{\partial T}\right|_{\rho_{0} \rho_{m}}}\left[1-\frac{\left.\frac{\partial \sigma}{\partial T}\right|_{\rho_{0} \rho_{m}}}{\left.\frac{\partial \sigma}{\partial T}\right|_{P_{1} \rho_{0}}}\right]\right\}
$$

Le dernier est un mode de diffusion thermique :

$$
\omega=-i k^{2} \frac{\chi}{\left.T \frac{\partial \sigma}{\partial T}\right|_{P \rho_{0}}} .
$$

Si nous regardons maintenant l'importance de ces différents modes dans le comportement des fonctions de corrélation de charge et de masse du SFS, nous constatons que la fonction de corrélation de charge [formule (51) dans [3]] est dominée par le premier mode, de diffusion de charge. Par contre la fonction de corrélation de masse [formule (34) dans [3]] est dominée par le mode sonore et le mode de diffusion thermique, dans les proportions comparables, exactement comme un fluide simple non chargé.
4. Conclusion. - Un tel système, formé de deux espèces ioniques de charges de signes contraires, a un comportement non coulombien, dans ce sens que tout gradient de charge, aussi faible soit-il, est résorbé dans un temps qui reste fini : $\left(4 \pi e^{2} \alpha\right)^{-1}$. Il est intéressant d'évaluer ce temps à partir de la conductivité électrique déterminée dans [3]. Nous obtenons pour $\mathrm{NaCl}\left(4 \pi e^{2} \alpha\right)^{-1}=4 \times 10^{-14} \mathrm{~s}$, temps comparable à l'inverse de la fréquence plasma, qui vaut alors $2 \times 10^{-14} \mathrm{~s}$. Ce processus rapide fait que le fluide se comporte comme un fluide simple non chargé.

Il est important de noter qu'aucun mode optique n'apparaît à cette limite. Ceci peut sembler contradictoire avec les résultats de dynamique moléculaire [3], où l'on observait un pic plasmon dans la fonction de corrélation de charge, et où, à l'inverse, aucun son 
n'apparaissait, même dans la fonction de corrélation de masse. Nous sommes amenés à penser que les valeurs de $k$ considérées dans [3] restaient trop grandes, et que le mode plasmon correspond à un domaine intermédiaire de $k$. Un examen attentif des courbes $\mathrm{du}$ facteur de structure dynamique de charge dans [3] montre, d'ailleurs, le caractère résonnant en $k$ de ce mode plasmon; la largeur du pic est la plus faible pour une certaine valeur de $k$ et augmente de part et d'autre. Pour des valeurs suffisamment faibles de $k$ les oscillations plasma sont complètement amorties par l'interdiffusion et l'on observe alors une oscillation sonore dans la fonction de corrélation de masse $\left({ }^{1}\right)$.

Enfin, nous tenons à souligner que ces résultats ne sont pas spécifiques au SFS ; ils restent qualitativement valables pour n'importe quel sel fondu bien que le couplage entre charge et masse introduise une certaine complication.

Remerciements. - Nous tenons à remercier J. P. Hansen pour les nombreuses et fructueuses discussions que nous avons eues avec lui.

\section{Bibliographie}

[1] Mountain, R. D. and Deutch, J. M., J. Chem. Phys. 50 (1969) 1103

[2] Vieillefosse, P. and Hansen, J. P., Phys. Rev. A 12 (1975) 1106.

[3] Hansen, J. P. and Mc Donald, I. R., Phys. Rev. A 11 (1975) 2111.

[4] LANDAU, L. et Lifchitz, E., Mécanique des Fluides $\$ 49$ (Editions Mir, Moscou)

$\left({ }^{1}\right)$ Remarquons que l'absence de mode optique aux petits $k$ ne peut être imputée au formalisme utilisé qui, rappelons-le [2], avait mis en évidence les oscillations plasma avec la bonne relation de dispersion pour le P.U.C. 